\title{
A $0535+26$ in the April 2010 outburst: probing the accretion regime at work
}

\author{
D. Müller ${ }^{1}$, D. Klochkov ${ }^{1}$, I. Caballero ${ }^{2}$, and A. Santangelo ${ }^{1}$ \\ ${ }^{1}$ Institut für Astronomie und Astrophysik, Universität Tübingen, Sand 1, 72076 Tübingen, Germany \\ e-mail: daniela.mueller@astro.uni-tuebingen.de \\ 2 Laboratoire AIM, CEA/IRFU, CNRS/INSU, Université Paris Diderot, CEA DSM/IRFU/SAp, 91191 Gif-sur-Yvette, France
}

Received 7 September 2012 / Accepted 5 March 2013

\section{ABSTRACT}

\begin{abstract}
Context. A number of accreting X-ray pulsars experience spectral changes, both on the long time scales and on the time scales of the neutron star spin period. The sources seem to form two distinct groups that differ by the type of the spectral variations with flux. Such a bimodality probably reflects two different regimes of accretion that may result in a particular pulsar depending on its luminosity so-called sub- and super-critical regimes.

Aims. We investigated the behavior of the spectral parameters of the Be/X-ray binary system A $0535+26$, as a function of flux and pulse phase.

Methods. We used the data collected with INTEGRAL and RXTE during the April 2010 outburst of the source. We analyzed the phase-averaged and phase-resolved spectra and performed pulse-to-pulse spectral analysis of the pulsar.

Results. Our analysis reveals variability in the continuum parameters of the source's pulse-averaged spectrum with flux. The pulseaveraged cyclotron line energy does not change with the source luminosity during the outburst, which is consistent with previous studies. Our pulse-phase resolved and pulse-to-pulse analyses reveal, however, indications for a positive correlation of the cyclotron line energy with flux, as well as a flux-dependence of the continuum parameters. Based on the observed behavior, we argue that A $0535+26$ operates at the sub-critical accretion regime.
\end{abstract}

Key words. X-rays: binaries - stars: neutron - accretion, accretion disks - pulsars: individual: A 0535+26

\section{Introduction}

The X-ray transient A $0535+26^{1}$ was discovered by the satellite Ariel $V$ during a giant outburst in April 1975 (Rosenberg et al. 1975). The source belongs to the class of Be/X-ray binaries. It consists of an accreting neutron star orbiting the O9.7 IIIe optical star HDE 245770 (Li et al. 1979; Giangrande et al. 1980). The system regularly undergoes X-ray outbursts separated by quiescence phases (Motch et al. 1991). In Be/X-ray binaries, outbursts are often divided into normal and giant ones. Normal outbursts repeat periodically at times near the periastron passage and are thought to originate from an increase of the accretion rate $\left(L_{\max } / L_{\min } \approx 100\right)$ due to a closer position of the neutron star to its Be star companion (Stella et al. 1986). Giant outbursts show a larger increase in luminosity $\left(L_{\max } / L_{\min } \approx 100-1000\right)$ and last several tens of days (Stella et al. 1986). Often there is, however, no strict distinction between the two types of outbursts. Thus, some outbursts in A $0535+26$ can not be clearly assigned to one of those two groups. Besides the outburst in 1975, A 0535+26 went through giant outbursts in 1980 (Nagase et al. 1982), 1983 (Sembay et al. 1990), 1989 (Makino et al. 1989), 1994 (Finger et al. 1996), 2005 (Tueller et al. 2005), 2009 (Krimm et al. 2009; Caballero et al. 2009, 2011c) and 2011 (Camero-Arranz et al. 2011; Tchernin et al. 2011; Caballero et al. 2011a).

The distance of the system has been measured to be around $2 \mathrm{kpc}$ (Steele et al. 1998). Recent measurements of the orbital

\footnotetext{
A $0535+26$ is referred to as $1 \mathrm{~A} 0535+262$ in SIMBAD Astronomical Database.
}

period give $\sim 111.1$ days (Finger et al. 2006). The pulse period is around 103.39s (Caballero et al. 2007).

The phase-averaged X-ray spectrum of A $0535+26$ can be described by a hard power law $E^{-\Gamma}(\Gamma=0.8-1.1$ for $2-18 \mathrm{keV}$ Ariel-V/SSI observations, Ricketts et al. 1975) modified above $\sim 15-20 \mathrm{keV}$ by an exponential roll-off (TTM/HEXE spectrum in 2-156 keV, Kendziorra et al. 1994). Alternatively, Acciari et al. (2011) used the thermal Comptonization model to fit the lowenergetic part of the X-ray continuum. Additionally, the spectrum shows absorption lines, interpreted as cyclotron resonant scattering features (CRSFs). Such features are caused by resonant scattering of photons off the electrons in Landau levels (see, e.g., Truemper et al. 1978; Isenberg et al. 1998). The energy of the fundamental line and the spacing between harmonics are directly proportional to the magnetic field strength. Two cyclotron absorption lines at about $50 \mathrm{keV}$ and $100 \mathrm{keV}$ were reported and interpreted as the fundamental and the first harmonic and implying a magnetic field strength of about $4.3 \times 10^{12} \mathrm{G}$ (Kendziorra et al. 1994; Caballero et al. 2007). While the presence of the fundamental line at about $50 \mathrm{keV}$ was initially questioned (Grove et al. 1995; Araya \& Harding 1996), newer analyses clearly confirm the presence of both lines and their interpretation as a fundamental cyclotron feature and its first harmonic (Caballero et al. 2007).

An evolution of the cyclotron line energy with the source luminosity has been reported for several accreting X-ray pulsars. A negative correlation of the line energy with luminosity has been observed in V $0332+53$ and 4U 0115+63 (Tsygankov et al. 2006, 2007, 2010; Mowlavi et al. 2006; for 4U0115+63 see, 
however, Müller et al. 2012). A positive correlation was detected for Her X-1 and GX 304-1 (Staubert et al. 2007; Klochkov et al. 2012). A similar behavior is seen in the pulse-to-pulse variations. For V $0332+53$ and $4 \mathrm{U} 0115+63$, a negative correlation of cyclotron line energy with the amplitude of individual pulses and a softening of the spectral continuum with pulse amplitude was reported, whereas for Her X-1 and A $0535+26$ a positive correlation of the line energy with pulse amplitude and a hardening of the spectrum with pulse amplitude was observed (Klochkov et al. 2011). Since A 0535+26 showed the spectral-flux dependence on the pulse-to-pulse time scales only, it is important to verify and study this behavior, using the data from a new outburst of the source.

Pulse-phase resolved analysis shows that the spectrum of the main peak of the pulse profile seems to be harder than the spectrum of the secondary peak (Kendziorra et al. 1994; Caballero 2009). Caballero (2009) also found a noticeably lower cyclotron line energy during the main peak compared to the secondary one.

In this work, we analyzed the April 2010 outburst of A 0535+26. On April 3 2010, a brightening of A 0535+26 in the Swift/BAT light curve was reported (Caballero et al. 2010). In the $20-100 \mathrm{keV}$ energy band of INTEGRAL/ISGRI the flux of the source reached a value of about $0.92 \mathrm{Crab}$ and the fundamental cyclotron line was detected at about $47.6 \mathrm{keV}$ (Caballero et al. 2010). The outburst analyzed in this work took place after the giant outburst of December 2009, which reached a flux of about $5.14 \mathrm{Crab}$ in the $15-50 \mathrm{keV} \mathrm{Swift/BAT}$ light curve (Caballero et al. 2010).

Our analysis is focused on the spectral evolution of A $0535+26$ as a function of luminosity. We analyzed phaseaveraged, pulse-phase resolved, and pulse-amplitude resolved (pulse-to-pulse) spectra of the 2010 outburst, to explore spectral variations on short (down to the pulsar's spin period) and long (several days) time scales. A description of the observations and data processing is given in Sect. 2. In Sect. 3, we describe the analysis of the phase-averaged, phase-resolved and pulse-topulse spectra. In Sects. 4 and 5, we discuss and summarize our results.

\section{Observations and data processing}

For our analysis, we used data from the JEM-X and IBIS instruments of the INTErnational Gamma-Ray Astrophysics Laboratory satellite (INTEGRAL, Winkler et al. 2003) and from the PCA and HEXTE instruments onboard Rossi X-ray Timing Explorer (RXTE, Bradt et al. 1993).

The JEM-X monitor provides spectra and images with arcminute resolution in the $3-35 \mathrm{keV}$ energy band (Lund et al. 2003). The high angular resolution gamma-ray imager IBIS consists of two detector layers, ISGRI and PICsIT (Ubertini et al. 2003). The ISGRI layer, which we used for our analysis, is sensitive at energies from $15 \mathrm{keV}$ to $1 \mathrm{MeV}$ (Lebrun et al. 2003). The PCA instrument onboard RXTE consists of a set of five proportional counters and is sensitive to energies $2-60 \mathrm{keV}$ (Jahoda et al. 2006). The high-energy detector HEXTE operates in the energy range 15-250 keV (Rothschild et al. 1998).

We used data covering the time from the maximum to the decay of the April 2010 outburst (2010-04-03 to 2010-04-17, Table 1). A Swift/BAT light curve of A $0535+26$ with the indicated observations is shown in Fig. 1.

The INTEGRAL data were processed using OSA 9.0. For the ISGRI data we performed an additional gain correction to account for known problems in the OSA 9.0 energy calibration. We used the JEM-X data in the 5-30 keV range and



Fig. 1. The Swift/BAT daily light curve of the April 2010 outburst of A $0535+26$. The INTEGRAL and RXTE observations which we used for our analysis are indicated with dashed (INTEGRAL) and solid lines (RXTE).

Table 1. Selected observations.

\begin{tabular}{lcc}
\hline \hline Observation & Exposure [ks] & Flux in Crab [10-100 keV] \\
\hline INTEGRAL 0912 & 65.1 & 0.978 \\
INTEGRAL 0914 & 23.8 & 0.675 \\
INTEGRAL 0915 & 28.6 & 0.368 \\
INTEGRAL 0916 & 29.4 & 0.191 \\
INTEGRAL 0917 & 31.7 & 0.096 \\
\hline RXTE 95347-02-01-00 & 3.3 & 0.908 \\
RXTE 95347-02-01-01 & 3.3 & 0.986 \\
RXTE 95347-02-01-02 & 3.0 & 0.715 \\
\hline
\end{tabular}

the ISGRI data in the $20-145 \mathrm{keV}$ range. We added systematic errors of $3 \%$ for JEM-X and $2 \%$ for ISGRI, which we estimated by fitting a Crab spectrum accumulated with the respective instruments with a canonical power-law with a photon index of $\sim 2.1$. The Crab spectra are taken during the same observations since both sources are present simultaneously in the INTEGRAL field-of-view.

We used HEASoft Version 6.11 and selected the energy ranges $5-60 \mathrm{keV}$ for PCA and $20-145 \mathrm{keV}$ for HEXTE for the RXTE data. In the PCA spectra we excluded data below $5 \mathrm{keV}$ due to an instrumental background feature at the xenon $L$ edge around $\sim 4.8 \mathrm{keV}$, which is poorly modeled and might introduce systematic effects to our spectral fits (Rothschild et al. 2006; Caballero et al. 2008) We added a systematic error of $1 \%$ for PCA and $1 \%$ for HEXTE data as recommended by the instruments teams. A systematic error for the HEXTE data is required due to the stop of the rocking motion of the HEXTE Cluster A in 2006 and HEXTE Cluster B in December 2009. Since then, HEXTE Cluster $A$ is fixed on-source while HEXTE Cluster $B$ measures the background, which is then converted to an adequate background for Cluster $A$ (Pottschmidt et al. 2006). Similarly to Rothschild et al. (2011), we used the recorn model in XSPEC to account for uncertainties in the correction factor of the background.

In order to select time intervals for the phase-resolved and pulse-to-pulse analyses, we extracted light curves for each observation. We then converted the photon arrival times to the solar system barycenter and corrected them for the orbital motion of the binary system using the ephemeris from Finger et al. (2006). The pulse period was determined with the epoch-folding technique. The pulse phase zero corresponds to the minimum in the pulse profile (see Fig. 8). 
D. Müller et al.: A $0535+26$ in the April 2010 outburst: probing the accretion regime at work

Table 2. Fit parameters for the INTEGRAL observations 0912, 0914, 0915, 0916, and 0917 and the RXTE observations 95347-02-01-00, 9534702-01-01, and 95347-02-01-02.

\begin{tabular}{lcccccc}
\hline \hline Observation & $\Gamma$ & $E_{\text {rolloff }}$ & $E_{\text {cyc }}$ & $\sigma_{\text {cyc }}$ & $\tau_{\text {cyc }}$ & $\chi_{\text {red }}^{2} /$ d.o.f. \\
\hline INTEGRAL 0912 & $0.56_{-0.06}^{+0.06}$ & $17.21_{-0.48}^{+0.44}$ & $44.82_{-0.37}^{+0.36}$ & $12.95_{-0.80}^{+0.92}$ & $13.16_{-1.60}^{+2.08}$ & $1.1 / 110$ \\
INTEGRAL 0914 & $0.30_{-0.13}^{+0.11}$ & $15.32_{-0.90}^{+0.77}$ & $43.04_{-0.51}^{+0.45}$ & $14.67_{-1.21}^{+1.51}$ & $24.29_{-4.37}^{+6.62}$ & $0.9 / 110$ \\
INTEGRAL 0915 & $0.65_{-0.07}^{+0.07}$ & $18.18_{-0.64}^{+0.64}$ & $43.99_{-0.41}^{+0.41}$ & $10.97_{-0.68}^{+0.75}$ & $16.18_{-1.70}^{+2.04}$ & $0.9 / 110$ \\
INTEGRAL 0916 & $0.83_{-0.09}^{+0.08}$ & $19.88_{-1.01}^{+1.00}$ & $44.31_{-0.67}^{+0.70}$ & $11.14_{-1.02}^{+1.18}$ & $16.82_{-2.47}^{+3.24}$ & $0.8 / 110$ \\
INTEGRAL 0917 & $1.01_{-0.09}^{+0.08}$ & $23.75_{-1.45}^{+1.59}$ & $45.83_{-0.97}^{+1.06}$ & $8.67_{-2.26}^{+1.22}$ & $14.40_{-2.26}^{+2.75}$ & $0.9 / 110$ \\
\hline RXTE 95347-02-01-00 & $0.44_{-0.02}^{+0.02}$ & $16.24_{-0.15}^{+0.15}$ & $44.24_{-0.29}^{+0.29}$ & $9.01_{-0.34}^{+0.36}$ & $8.69_{-0.49}^{+0.52}$ & $1.2 / 152$ \\
RXTE 95347-02-01-01 & $0.43_{-0.02}^{+0.02}$ & $15.95_{-0.15}^{+0.15}$ & $43.92_{-0.29}^{+0.29}$ & $9.80_{-0.34}^{+0.39}$ & $9.28_{-0.55}^{+0.59}$ & $1.5 / 152$ \\
RXTE 95347-02-01-02 & $0.47_{-0.02}^{+0.02}$ & $16.26_{-0.18}^{+0.18}$ & $43.31_{-0.33}^{+0.33}$ & $9.16_{-0.40}^{+0.41}$ & $9.00_{-0.59}^{+0.63}$ & $1.3 / 152$ \\
\hline
\end{tabular}

Notes. The errors are at $1 \sigma$ confidence level.

a)

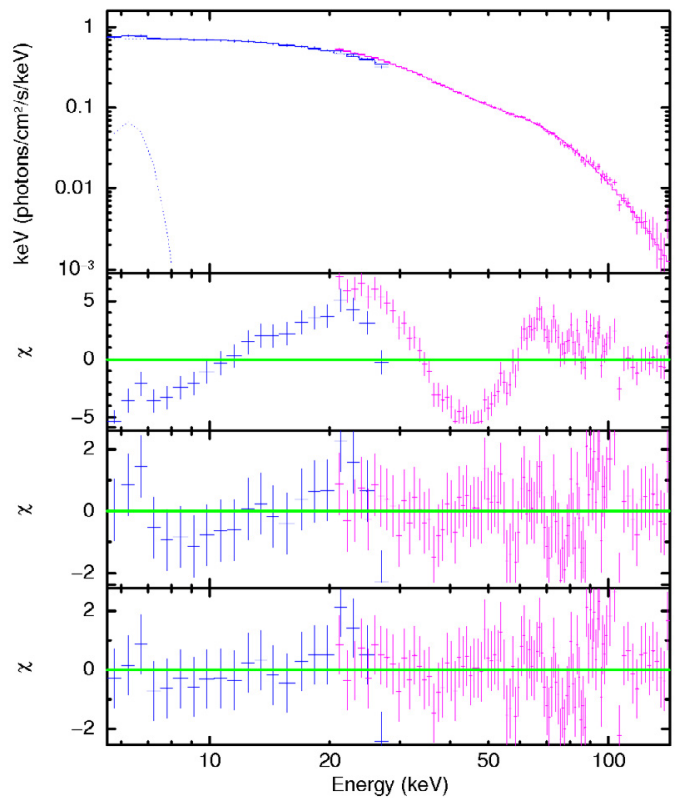

Fig. 2. Unfolded spectrum of A $0535+26$ from the INTEGRAL revolution 0912 a), the residuals of the fit with the model without the $6.4 \mathrm{keV}$ Fe emission and the cyclotron absorption lines b), the residuals after inclusion in the model of the cyclotron absorption line $\mathbf{c}$ ) and the Fe emission line d).

\section{Spectral analysis}

\subsection{Phase averaged spectra}

The spectra of A $0535+26$ for various luminosity states were best fitted by a power-law with a photon index $\Gamma$ modified by an exponential rolloff with the folding energy $E_{\text {rolloff }}$ (the cutoffpl model in XSPEC):

$$
F(E)=\text { const. } E^{-\Gamma} e^{-E / E_{\text {rolloff }}} .
$$

We included an emission line at around $6.4 \mathrm{keV}$ to model the $\mathrm{Fe}$ $\mathrm{K} \alpha$ fluorescence line. Additionally, the residuals of the fit require inclusion of a an absorption line around $40 \mathrm{keV}$ (Fig. 2) which we interpret as a CRSF (see Sect. 1). To account for this feature, we included a multiplicative absorption line with a Gaussian optical depth profile (the XSPEC gabs model) with a centroid energy of $\sim 44.4 \mathrm{keV}$ :

$F(E)=\exp \left[-\frac{\tau_{\mathrm{cyc}}}{\sqrt{2 \pi} \sigma_{\mathrm{cyc}}} \exp \left(-\frac{\left(E-E_{\mathrm{cyc}}\right)^{2}}{2 \sigma_{\mathrm{cyc}}^{2}}\right)\right]$,


Fig. 3. Photon index $\Gamma$, rolloff energy $E_{\text {rolloff }}$ and cyclotron line energy $E_{\text {cyc }}$ as a function of the source flux in the $10-100 \mathrm{keV}$ band in units of $\mathrm{Crab}$ and the luminosity in erg/s for a distance of $2 \mathrm{kpc}$ (upper $X$-axis). The INTEGRAL observations are marked with circles, the RXTE observations - with triangles. The errors are at $1 \sigma$ confidence level.

where $E_{\text {cyc }}$ is the cyclotron line centroid energy, $\tau_{\text {cyc }}$ and $\sigma_{\text {cyc }}$ characterize the line strength and width respectively. The RXTE observations also indicate the presence of the first harmonic line around $104 \mathrm{keV}$. The best-fit parameters for the INTEGRAL and RXTE observations are summarized in Table 2. Figure 3 shows the photon index $\Gamma$, the rolloff energy $E_{\text {rolloff }}$ and the cyclotron line energy $E_{\text {cyc }}$ as a function of $10-100 \mathrm{keV}$ flux in Crab units. The latter is obtained from a direct comparison of the source flux with that of the simultaneously observed Crab nebula. Because the first harmonic in the RXTE spectra is not statistically significant and to be consistent with the INTEGRAL spectral model, only one Gaussian absorption line was included in our spectral model, accounting for the fundamental cyclotron line.

The photon index decreases from $\sim 1.0$ for low luminosities to $\sim 0.5$ for the outburst maximum, indicating a hardening of the power-law continuum with flux. The rolloff energy $E_{\text {rolloff }}$, however, decreases from $\sim 24$ to $\sim 16 \mathrm{keV}$ from the lowest to highest luminosity. That is, the part of the continuum around $E_{\text {rolloff }}$ softens with flux. No correlation of the cyclotron line energy 


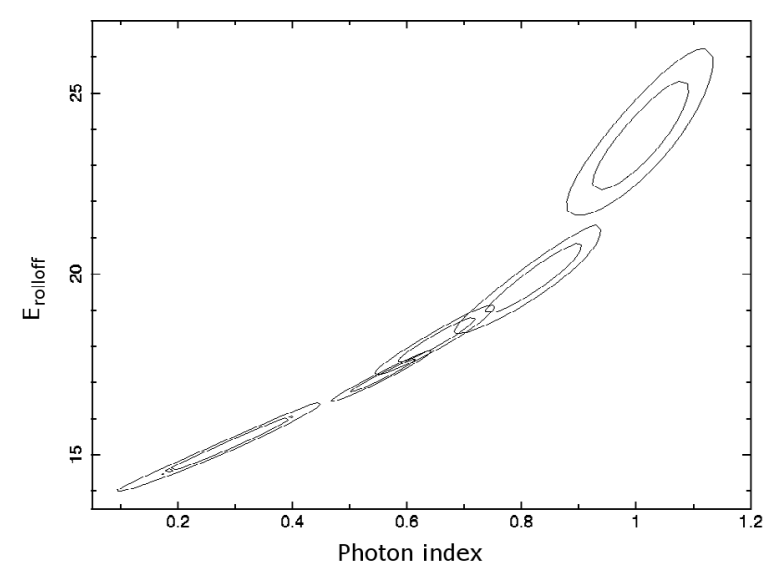

Fig. 4. Contour plot of the folding energy $E_{\text {rolloff }}$ and the photon index $\Gamma$ for all five INTEGRAL observations. The contour levels are at $\chi_{\min }^{2}+1.0$ (corresponds to 68\% confidence for one chosen parameters) and $\chi_{\min }^{2}+$ 2.3 (corresponds to $68 \%$ confidence for two chosen parameters).

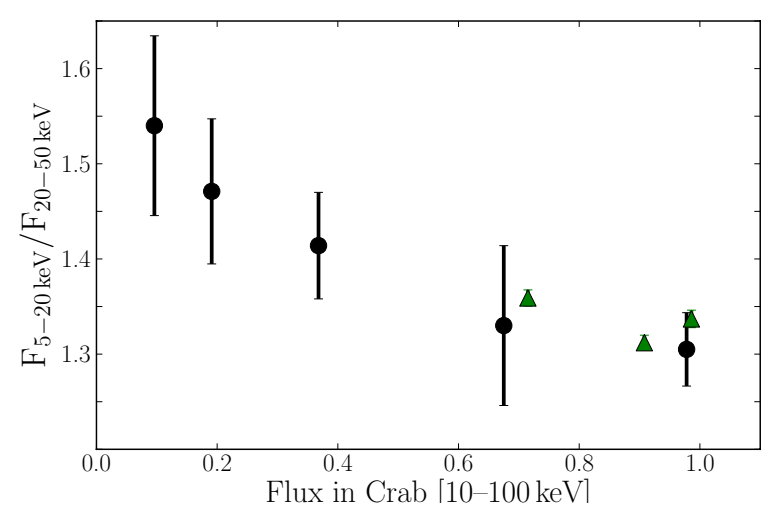

Fig. 5. 5-20 keV to $20-50 \mathrm{keV}$ flux ratio as a function of the source flux in units of Crab. The graph indicates a hardening the spectrum with flux.

with the source luminosity is observed. The cyclotron line energy, however, varies irregularly between $\sim 43 \mathrm{keV}$ and $\sim 46 \mathrm{keV}$. To rule out any coupling between $E_{\text {rolloff }}$ and $\Gamma$, we produced contour plots of those two parameters (Fig. 4). Since the evolution of $\Gamma$ indicates a hardening of the spectrum with increasing flux while the variation of $E_{\text {rolloff }}$ suggests the opposite behavior, we calculated the hardness ratio to study the spectral variations independently of the spectral model. Figure 5 shows a ratio of the $5-20 \mathrm{keV}$ flux to the $20-50 \mathrm{keV}$ flux as a function of the $10-100 \mathrm{keV}$ flux. The observed decrease of the ratio implies an overall hardening of the spectrum with flux. A similar behavior is reported in Caballero et al. (2013) for the normal double-peaked outburst of August 2009. The results of this analysis, i.e. no correlation for the cyclotron line energy and a spectral hardening with flux, seem to support our results in the analyzed luminosity range. Following Acciari et al. (2011), we tried to model the spectra with the compTT model in XSPEC. We find that although the compTT model provides a good description of the X-ray spectrum below $\sim 35 \mathrm{keV}$ (the energy range used in Acciari et al. 2011), it produces strong residuals when applied to the overall energy range evaluated in this paper, thus making the compTT overall fit formally unacceptable. Nevertheless, we analyzed the evolution of the cyclotron line energy as a function of flux using compTT and found it to be consistent with the results, obtained with our phenomenological model.

\subsection{Pulse-phase resolved spectroscopy}

To select pulse-phase intervals, we produced pulse profiles which are similar to those reported earlier (e.g. Caballero 2009 and Caballero et al. 2011b) and do not show strong variation with flux. We then extracted spectra in 10 equal phase bins for each observation. That is, we performed pulse-phase resolved spectroscopy separately for different flux levels. Figure 6 summarizes the evolution of the photon index and the cyclotron line energy with pulse phase at different fluxes. For each pulse-phase bin separated by vertical dashed lines and marked by a number from 1 to 10 , left column, $\Gamma$ and $E_{\text {cyc }}$ are shown as a function of $10-100 \mathrm{keV}$ flux in the middle and the right column, respectively. To increase the photon statistics in the observations at the lowest flux levels, we summed up the observations from the INTEGRAL revolutions 0916 and 0917. For each pulse-phase bin, we performed a linear fit of the $\Gamma$-flux and $E_{\text {cyc }}$-flux dependencies shown by the solid lines. The slopes of the linear fits with the corresponding $1 \sigma$ uncertainties are shown in the bottom panels of the left column. The value of the slope with the associated uncertainty gives a rough estimate of the significance of the linear correlation between the parameters.

$\Gamma$ and $E_{\text {cyc }}$ show systematic variations with pulse phase and flux. The evolution of $\Gamma$ as a function of flux, for individual pulse-phase intervals, indicates a hardening of the power-law part of the continuum with flux in almost all phase bins. In the pulse phases between 0.7 and 1.0 there is a strong indication (slope/error $\geq 3 \sigma$ ) for a positive correlation of the cyclotron line energy $E_{\text {cyc }}$ with flux (bottom panel of left column in Fig. 6). To estimate quantitatively the significance of the non-zero slopes, we performed a F-test, which yields a value $P$ of the probability that the improvement in $\chi^{2}$ obtained by moving from a fit of the dependences by a constant to a linear fit with non-zero slope is due to statistical flucuations. Lower values of $P$, thus, indicate larger significance. In case of the $\Gamma$-flux dependence, the $P$-values for most phase bins are at the level of a few per cent indicating marginal significance for individual bins. For the cyclotron line energy dependence on flux in the phase interval $0.7-1.0$, i.e. the phase bins 8,9 , and 10 , the $P$-values are 0.027 , 0.009 , and 0.001 , respectively.

To check possible influence of any systematic differences in calibration between the INTEGRAL and RXTE instruments on our results, we re-calculated the significances using only the INTEGRAL data points. For the dependence of $\Gamma$ on flux, the $P$-values remained at a level of a few per cent. For the $E$-flux dependence in the phase bins 8,9 , and 10 , the $P$-values are found to be $0.08,0.17$, and 0.05 , respectively. The values for the slope, however, did not change considerably. We find, therefore, no indication that the measured correlations are driven by systematic differences between the instruments onboard the two satellites.

As before, to check the stability of our results with respect to the choice of the continuum model, following Acciari et al. (2011) we fitted the spectra using the thermal Comptonization model compTT in XSPEC. Even though the spectral fits are formally unacceptable (similar to the case of phase-averaged analysis, see Sect. 3.1), the evolution of the cyclotron line energy with flux is well reproduced.

In the following, the two peaks of the double-peaked pulse profile are referred to as the main and secondary pulse. To characterize spectral variations within larger pulse-phase intervals, namely in the main and secondary pulses, we extracted spectra for two broad pulse-phase intervals for each observation. The first interval, $0.1-0.5$, contains the main peak, the second one, $0.6-1.0,-$ the secondary peak. The best-fit parameters, as a 

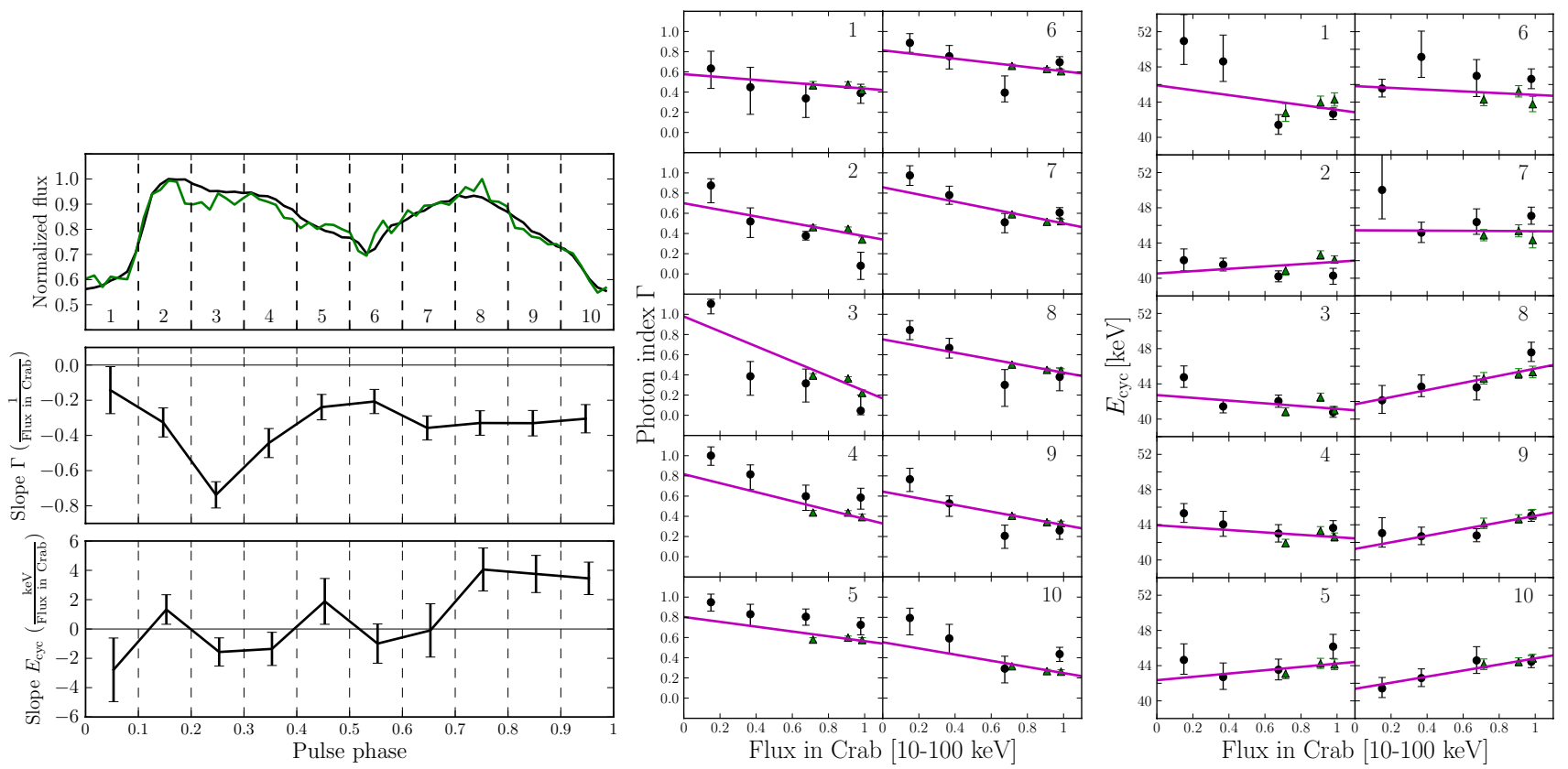

Fig. 6. Best-fit parameters of the pulse-phase resolved spectra at different flux levels with $1 \sigma$ errors. Left column: in the top panel, the averaged and normalized pulse profile of the INTEGRAL observation during rev. 0912 (3-80 keV, black line) and the RXTE observation 95347-02-0101 (3-60 keV, green line) are shown. The pulse-phase intervals for which the spectra were extracted are indicated by vertical dashed lines and numbered from "1" to "10". Within each bin, we analyzed the spectral parameters as a function of flux. The middle and bottom panels represent the slopes with associated $1 \sigma$-uncertainties obtained from the linear fits to the $\Gamma$-flux and $E_{\text {cyc }}$-flux dependencies in each pulse-phase bin which are shown in the middle and right columns. Middle and right columns: the photon index $\Gamma$ and the cyclotron line energy $E_{\mathrm{cyc}}$ as a function of the $10-100 \mathrm{keV}$ flux for each of the ten phase intervals marked by the numbers. The solid lines show the linear fits to the data points. The RXTE observations are marked with triangles, while the INTEGRAL observations - with circles.

function of flux, for the two phase intervals are shown in Fig. 7. To characterize the hardness of the continuum by the photon in$\operatorname{dex} \Gamma$ only, we fixed the rolloff energy $E_{\text {rolloff }}$ to its averaged value of $16 \mathrm{keV}$ for the upper plot of Fig. 7. The spectrum is harder and the cyclotron line energy lower for the main pulse. We observe an indication for a spectral hardening and a positive trend of the cyclotron line energy with flux for the secondary peak. The values for the slopes of the linear fit together with the results of the linear correlation analysis are shown in Table 3.

\subsection{Pulse-to-pulse analysis}

We performed a spectral analysis of the source on a pulse-topulse basis, similar to Klochkov et al. (2011). To identify individual pulsations, we extracted light curves for all INTEGRAL and RXTE observations. Following Klochkov et al. (2011), we have chosen the secondary peak of the pulse profile (Fig. 8) for our analysis, to be able to compare our results. In the pulse profiles above $\sim 20-30 \mathrm{keV}$ the secondary peak is higher than the first one, thus, providing better statistics for the measurements of the cyclotron line around $50 \mathrm{keV}$. For each individual pulsation cycle, we calculated the mean ISGRI (for the INTEGRAL observations) and PCA (for the RXTE observations) count rate in the selected pulse-phase interval, which we refer to as height or amplitude of individual pulses. We then grouped pulses of a similar pulse height together to accumulate spectra as a function of pulse height.

For the pulse-to-pulse analysis of the INTEGRAL observations, we only used the data from the revolution 0912 taken during the maximum of the outburst, which provided the best photon statistics. Strong pulse-to-pulse variability can be seen clearly in Fig. 9, where the 20-120 keV ISGRI light curve, together with the repeated averaged pulse profile and the selected


Fig. 7. Best-fit spectral parameters as a function of flux for the main peak (phase interval 0.1-0.5) and the secondary peak (phase interval $0.6-1.0$ ) of the pulse profile. The data points and the linear fit (solid line) corresponding to the main peak are shown in black. The data corresponding to the secondary peak are shown in magenta. As in previous figures, the INTEGRAL observations are marked with circles, the RXTE observations - with triangles. For the upper plot, $E_{\text {rolloff }}$ was fixed to $16.0 \mathrm{keV}$ (see text). For the bottom plot all fit parameters are kept free. The corresponding values for the linear fit and the significance are shown in Table 3. 
Table 3. Slopes of the linear fits to the dependence of the spectral parameters on flux with the corresponding Pearson correlation coefficients and the associated two-sided null-hypotheses probabilities (a lower value indicates higher significance of the correlation).

\begin{tabular}{|c|c|c|c|c|}
\hline Observation & Parameter & Linear fit & Correlation coeff. & Probability \\
\hline \multicolumn{5}{|c|}{ Pulse-phase-resolved analysis } \\
\hline \multirow[t]{2}{*}{ phase $0.1-0.5$} & $\Gamma$ & $-0.00 \pm 0.03(\text { Crab unit })^{-1}$ & $\sim 0.28$ & $\sim 0.51$ \\
\hline & $E_{\text {cyc }}$ & 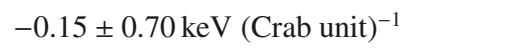 & $\sim-0.57$ & $\sim 0.14$ \\
\hline \multirow[t]{2}{*}{ phase $0.6-1.0$} & $\Gamma$ & $-0.12 \pm 0.03(\text { Crab unit })^{-1}$ & $\sim-0.83$ & $\sim 0.01$ \\
\hline & $E_{\mathrm{cyc}}$ & $2.09 \pm 0.95 \mathrm{keV}(\text { Crab unit })^{-1}$ & $\sim 0.60$ & $\sim 0.12$ \\
\hline \multicolumn{5}{|c|}{ Pulse-to-pulse analysis } \\
\hline \multirow[t]{2}{*}{ INTEGRAL } & $\Gamma$ & $(-5.79 \pm 1.66) \times 10^{-4} /($ ISGRI cts/s $)$ & $\sim-0.96$ & $\sim 0.01$ \\
\hline & $E_{\mathrm{cyc}}$ & $(0.69 \pm 6.78) \times 10^{-3} \mathrm{keV} /($ ISGRI cts/s $)$ & $\sim-0.17$ & $\sim 0.78$ \\
\hline \multirow[t]{2}{*}{ RXTE } & $\Gamma$ & $(-9.12 \pm 1.84) \times 10^{-5} /(\mathrm{PCA} \mathrm{cts} / \mathrm{s})$ & $\sim-0.99$ & $\sim 6.89 \times 10^{-4}$ \\
\hline & $E_{\mathrm{cyc}}$ & $(2.02 \pm 1.26) \times 10^{-3} \mathrm{keV} /(\mathrm{PCA} \mathrm{cts} / \mathrm{s})$ & $\sim 0.78$ & $\sim 0.12$ \\
\hline \multicolumn{5}{|c|}{ values from Klochkov et al. (2011) } \\
\hline \multirow[t]{2}{*}{ INTEGRAL } & $\Gamma$ & $(-8.71 \pm 0.86) \times 10^{-2} /($ ISGRI cts/s $)$ & -0.96 & 0.01 \\
\hline & $E_{\mathrm{cyc}}$ & $(1.36 \pm 0.53) \times 10^{-2} \mathrm{keV} /($ ISGRI cts/s $)$ & +0.94 & 0.02 \\
\hline \multirow[t]{2}{*}{ RXTE } & $\Gamma$ & $(-1.54 \pm 0.19) \times 10^{-4} /(\mathrm{PCA} \mathrm{cts} / \mathrm{s})$ & -0.98 & $4 \times 10^{-3}$ \\
\hline & $E_{\text {cyc }}$ & $(1.92 \pm 0.92) \times 10^{-3} \mathrm{keV} /($ PCA cts $/ \mathrm{s})$ & +0.70 & 0.19 \\
\hline
\end{tabular}

Notes. The uncertainties for the slopes are at $1 \sigma$ confidence level. The parameters from the pulse-phase resolved analysis of the phase intervals $0.1-0.5$ (main peak) and $0.6-1.0$ (secondary peak) are shown in the top section (for $\Gamma, E_{\text {rolloff }}$ was fixed to $16 \mathrm{keV}$ ). In the bottom section, the values from the pulse-to-pulse analysis are shown. For the fit of $\Gamma$ we fixed $E_{\text {rolloff }}=15.5 \mathrm{keV}$ for INTEGRAL and $E_{\text {rolloff }}=15.2 \mathrm{keV}$ for RXTE. For comparison, the values from Klochkov et al. (2011) are also shown.

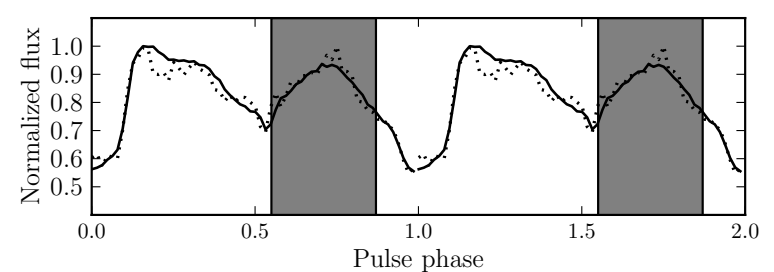

Fig. 8. Averaged pulse profile accumulated with INTEGRAL during its revolution 0912 (3-80 keV, continuous line) and with RXTE in the observation 95347-02-01-01 (3-60 keV, dotted line). The shaded area indicates the pulse-phase interval used for the pulse-to-pulse analysis.

pulse phase interval, is shown. The distribution of the measured amplitudes of individual pulses for this observation is shown in the left panel of Fig. 10. We accumulated spectra in five pulse-height bins: 100-210, 210-235, 235-260, 260-290, and 290-450 ISGRI counts/s.

For RXTE, we analyzed all three observations together as they were performed at a similar luminosity levels, close to the maximum of the source's outburst. The selected pulse height intervals for which the spectra have been accumulated are 850-1040, 1040-1210, 1210-1310, 1310-1480, and 1480-1850 PCA counts/s. The distribution of pulse heights for the RXTE data is shown in the right panel of Fig. 10.

The INTEGRAL and RXTE observations were analyzed separately because of possible systematic effects of non-perfect cross-calibration between the two observatories. The best-fit photon index and cyclotron line energy as a function of pulse height are shown in Fig. 11. We fixed the rolloff energy $E_{\text {rolloff }}$ to $15.5 \mathrm{keV}$ for the INTEGRAL and $15.2 \mathrm{keV}$, for the RXTE data for the plot of the photon index $\Gamma$. A significant decrease of the photon index (hardening of the power-law continuum) with pulse height is indicated by the data from both satellites. The cyclotron line energy, however, does not show any considerable evolution with pulse amplitude in the INTEGRAL data



Fig. 9. 20-120 keV ISGRI light curve of the INTEGRAL observation during rev. 0912 (black data points) together with the overplotted repeated averaged pulse profile (solid curve). The selected pulse-phase interval is indicated by shaded areas. The pulse-to-pulse variability can clearly be seen. The measured amplitudes of individual pulses (see text) are shown with magenta circles.

and an indication of a positive correlation with pulse height in the RXTE data. The results of the corresponding formal linear correlation analysis are shown in Table 3. Although the statistics of our data does not allow us to confirm the positive correlation between $E_{\text {cyc }}$ and pulse amplitude reported in Klochkov et al. (2011) (although, some support to the correlation is leaned by the RXTE data), the measured slopes are in agreement, within uncertainties, with those reported by Klochkov et al. (2011) and repeated in the bottom section of Table 3

\section{Discussion}

We have analyzed the phase-averaged, phase-resolved and pulseto-pulse spectra of A $0535+26$ as a function of the source luminosity. In all types of the analysis, the continuum gets harder with increasing flux. In some pulse-phase intervals, the data 

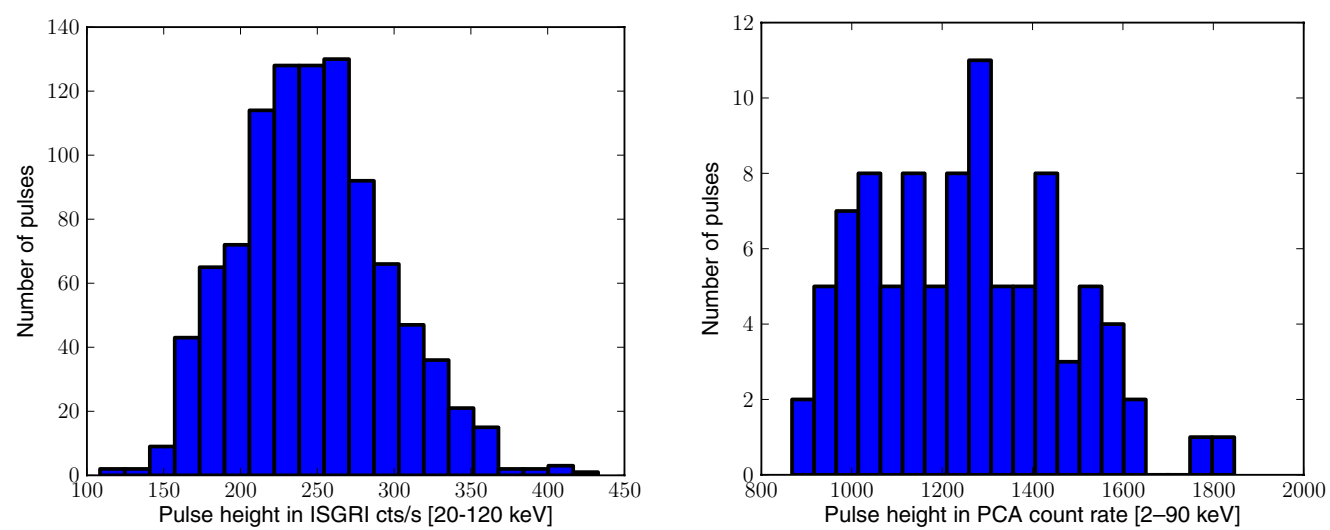

Fig. 10. Distributions of individual pulse heights measured with INTEGRAL (left) and RXTE (right).
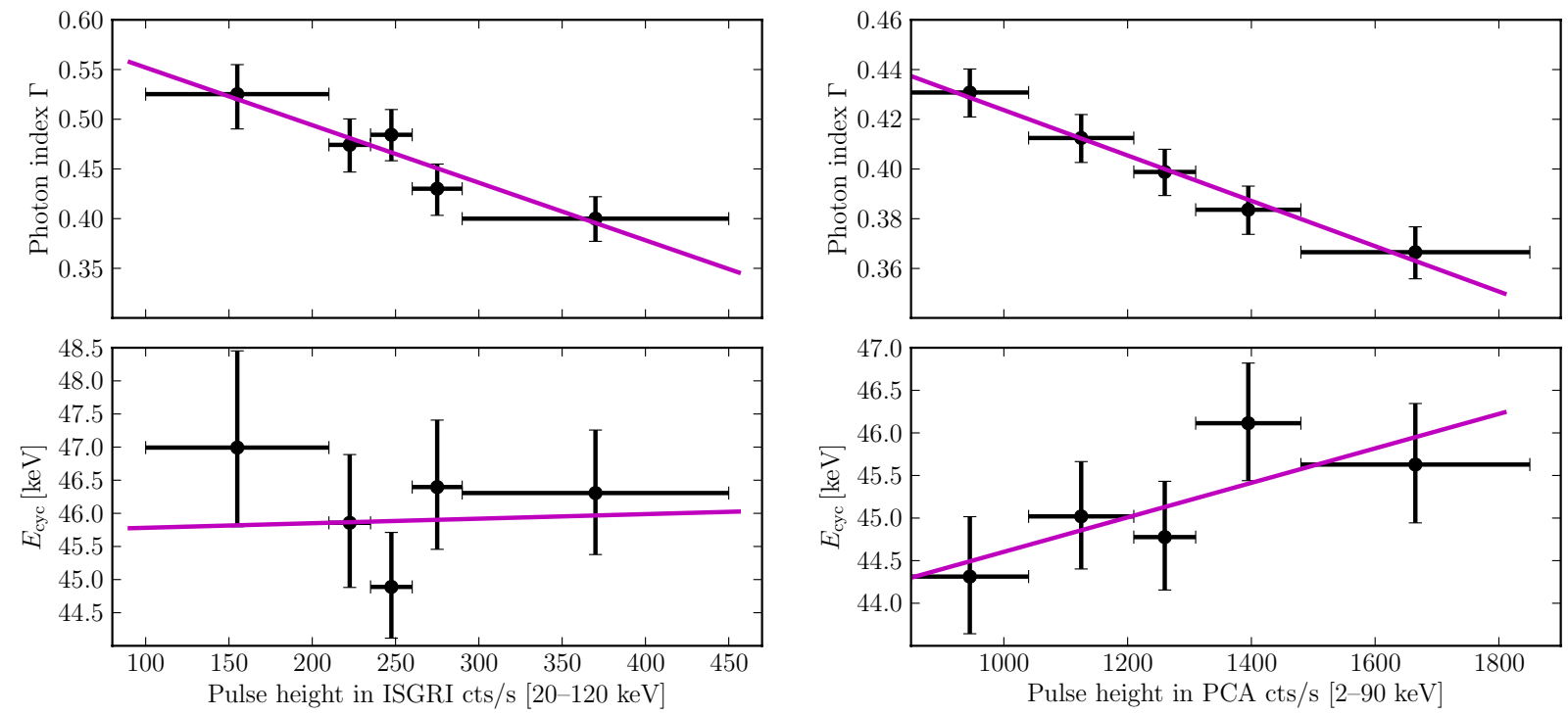

Fig. 11. Left: results of the pulse-to-pulse analysis of the INTEGRAL observation of rev. 0912. The photon index $\Gamma$ with fixed $E_{\text {rolloff }}=15.5 \mathrm{keV}$ and the cyclotron line energy $E_{\mathrm{cyc}}$ are plotted as a function of the individual pulse height. The vertical error bars indicate $1 \sigma$-uncertainties. The horizontal error bars correspond to the pulse-height bins used to accumulate the spectra. The solid lines indicate linear fits to the data. Right: same but for the RXTE observations. In the data for the upper panel $E_{\text {rolloff }}$ was fixes to $15.2 \mathrm{keV}$.

show indications of a positive $E_{\text {cyc }}$-flux correlation, although with marginal significance $(\sim 3 \sigma)$. The pulse-averaged centroid energy of the cyclotron line measured over longer time scales (days) shows some irregular variability but no clear dependence on flux, which is consistent with previous observations of this source. In the following, we discuss our results in more detail.

\subsection{Long-term spectral variations with luminosity}

As mentioned in the introduction, a relation between the cyclotron line centroid energy and luminosity was reported for a number of accreting pulsars. Since the work of Basko \& Sunyaev (1976), it is known that the geometry of the emitting structure (column), on/above the polar caps of an accreting neutron star, must be different for the luminosities above and below a certain critical value $L_{\mathrm{c}}$ which depends on the pulsar's properties. In the following, accreting pulsars with luminosities below $L_{\mathrm{c}}$ are referred to as sub-critical sources while those with luminosities exceeding $L_{\mathrm{c}}-$ as super-critical. According to this picture, $\mathrm{V} \mathrm{0332+53} \mathrm{and} \mathrm{4U0115+63} \mathrm{(for} \mathrm{4U0115+63} \mathrm{see,} \mathrm{however,}$ Müller et al. 2012), which show a negative $E_{\text {cyc }}-L$ correlation and a softening of the spectrum with flux, belong to so-called super-critical sources, whose luminosities are above $L_{\mathrm{c}}$. In such sources, infalling matter is most probably decelerated in a radiative shock, whose height is believed to increase with $L$, i.e., drift towards an area with a lower $B$-field strength. Her X-1 and GX 304-1, where a positive $E_{\text {cyc }}-L$ correlation and a hardening of the spectrum with flux are observed, are the examples of subcritical sources $\left(L<L_{\mathrm{c}}\right)$, where infalling matter is stopped by the Coulomb drag and collective plasma effects, rather than in a radiative shock. In such sources, the height of the emitting region decreases with increasing luminosity, owing to a corresponding increase in ram pressure of the infalling material (Staubert et al. 2007 and Becker et al. 2012).

The newest theoretical calculations by Becker et al. (2012) predict for A $0535+26$, a value of the critical luminosity $L_{\mathrm{c}} \sim$ $6.78 \times 10^{37} \mathrm{erg} / \mathrm{s}$, which is $\sim 5-6$ times larger than the peak flux of the outburst analyzed here (see the upper $X$-axis of Fig. 3 ). Thus, A $0535+26$ must have been accreting in a sub-critical regime during the outburst. Though, the key indication of this regime, namely the positive correlation between the CRSF energy and flux, is not firmly established in this source. Its X-ray continuum shows, however, a hardening with increasing flux, which is also observed in the other two sub-critical sources, Her X-1 and GX 304-1 (the super-critical sources V 0332+53 and 4U0115+63 show an opposite behavior of the spectral 
continuum, see above). If the accretion regime in A $0535+26$ is similar to one in Her X-1 and GX 304-1, the expected positive $E_{\text {cyc }}-L$ correlation must be smeared due to a special geometry of the emitting structure and/or a special orientation of the system with respect to the observer's line of sight. Alternatively, A 0535+26 might represent yet another, "third" accretion regime which yields very little, if any, variations in the height of the emitting region above the star surface (i.e., in the measured cyclotron line energy) in the observed range of luminosities. Indeed, while the luminosities of Her X-1 and GX304-1 are below, but relatively close to the corresponding values of $L_{\mathrm{c}}$ (see Fig. 2 in Becker et al. 2012), A 0535+26 is far below $L_{\mathrm{c}}$. Thus, the configuration of the accretion column in this source might differ substantially from those in the other two sub-critical pulsars. These considerations seem to be supported by the latest observations of a giant outburst of A $0535+26$, analyzed by Caballero et al. (2012), when the source luminosity approached $L_{\mathrm{c}}$ (i.e., a luminosity range of Her X-1 and GX 304-1). In this preliminary analysis, a few measurements of $E_{\mathrm{cyc}}$, corresponding to the highest luminosities, indeed show an indication of an increase towards higher fluxes (Fig. 1 of Caballero et al. 2012). Further observations are, however, necessary to confirm this effect.

\subsection{Pulse-phase dependence of the spectrum}

It is necessary to reconstruct the geometry of the rotating neutron star and its emitting regions to understand the results of the phase-resolved spectral analysis. Such a reconstruction can be made based on the observed pulse profiles of a pulsar using, e.g., the decomposition method by Kraus et al. (1995). This technique has recently been applied to A $0535+26$ by Caballero et al. (2011b). As a result, the pulse profiles of the source were reproduced in a model, including a hollow column, a thermal halo and taking into account relativistic light deflection in the strong gravitational field of the neutron star. Derived from the results of Caballero et al. (2011b), the observed pulse profile can be produced by one magnetic pole passing through the observer's line of sight, whereas the second pole is never seen directly from the top but only through the gravitationally bent radiation from the lateral emission of the accretion column and its thermal scattering halo.

Since we observed similar pulse profiles as those used by Caballero et al. (2011b), we adopt the reconstructed geometry for our data. In our analysis, we observe a slightly harder spectrum for the main pulse with respect to the secondary pulse. Magnetized plasma gets optically thin for photons $\left(E<E_{\text {cyc }}\right)$ which propagate parallel to the magnetic field lines. Thus, the main peak is probably formed by harder photons escaping the accretion column from top, along the field lines. About half a phase later, we probably see the lateral emission of both the accretion column and their thermal halos.

In our phase-resolved analysis, we observe variations of the cyclotron line energy with pulse phase in range $\sim 42-50 \mathrm{keV}$, which is probably caused by the changing viewing conditions of the scattering region and a dependence of the magnetic scattering cross sections on the angle between the magnetic field and the line of sight (Harding \& Daugherty 1991). Variations over pulse phase are also seen in the slope of the linear fits to the $E_{\text {cyc }}$ and $\Gamma$ values as a function of flux (Fig. 6). While a significant decrease of $\Gamma$ with flux is present at most pulse phases, a considerable (positive) $E_{\text {cyc }}$-flux correlation is only seen during the secondary peak (Figs. 6 and 7). According to the geometrical picture described above, the emerging radiation is emitted roughly perpendicular to the axis of the accretion column(s), that is, perpendicular to the field lines, in the secondary peak. As discussed in e.g. Ferrigno et al. (2011), at such angle, the resonant scattering features are expected to be deeper and narrower. Therefore, in the secondary peak, the viewing conditions of the line-forming region might be favorable for measuring $E_{\mathrm{cyc}}$ variations with flux. In the pulse-averaged spectral analysis, the dependence is smeared out.

\subsection{Pulse-to-pulse variability}

As we noted in Sect. 3.3, the results of our pulse-to-pulse analysis, namely the spectral variations with the amplitude of individual pulses, are in agreement with those reported by Klochkov et al. (2011) on the basis of the observations of the source's outburst in 2005 with better photon statistics. With our data, we have reproduced the hardening of the power-law with pulse amplitude. An indication of a positive correlation of the cyclotron line energy with flux can only be seen in the RXTE data (Fig. 11). If the positive correlation between the line energy and flux on the pulse-to-pulse time scale in A $0535+26$ is real, the question remains: why the correlation is not observed on the pulse-averaged spectra accumulated over longer time scales? A qualitative explanation of this discrepancy might be the following. Strong pulse-to-pulse variability of the source's flux leads to the relatively broad distribution of the amplitudes of individual pulses - more than a factor of two, as indicated in Fig. 10. Thus, pulse-averaged spectra accumulated over a longer time include contributions by pulses of very different heights. The pulse-topulse variability of the source's spectrum must lead to a substantial smearing effect, especially in case of a line-like feature. If A $0535+26$ indeed accrets in a regime with very little (if any) variation in the height of the emitting region with flux, as discussed in Sect. 4.1, the reduced sensitivity of the pulse-averaged spectroscopy to the $E_{\text {cyc }}$-flux correlation might indeed lead to the observed discrepancy between the pulse-averaged and pulse-topulse results.

\section{Summary and conclusions}

In this work, we present the spectral analysis of the observational data taken with RXTE and INTEGRAL on the Be/X-ray binary system A $0535+26$, during its outburst in April 2010. We studied the source's broad-band X-ray spectrum as a function of luminosity on the pulse-averaged, pulse-phase resolved, and pulseto-pulse basis. In all cases, a hardening of the X-ray continuum with flux is observed. This behavior is similar to that of other sub-critical sources, Her X-1 and GX 304-1. An indication of a positive correlation between the cyclotron line energy and flux (a characteristic feature of a sub-critical source) is only found in some pulse-phase resolved and pulse-to-pulse spectra (Fig. 6 and Table 3).

Based on the spectrum-flux dependence of A 0535+26, which is similar to that of other sub-critical sources, and on the source's luminosity, which is well below the critical luminosity $L_{\mathrm{c}}$, we conclude that the pulsar operates in a sub-critical accretion regime, where the radiative shock, if formed, is not capable of decelerating the accreted plasma to rest. Instead, the infalling matter is most probably stopped by the Coulomb drag and collective plasma effects. A similar behavior was reported in earlier works on this source, see e.g. Caballero et al. (2007, 2008, 2011c).

The lack of a firm detection of a $E_{\mathrm{cyc}}-$ flux correlation, which is expected in this accretion regime, we tentatively attribute to 
a substantially lower ratio of the source luminosity to the critical one, compared to other sub-critical sources. At such a low luminosity level, the amplitude of variation in the height of the line-forming region with luminosity (mass accretion rate) might be substantially narrower than that in Her X-1 and GX 304-1.

Acknowledgements. This research is based on observations with INTEGRAL, an ESA project with instruments and science data centre funded by ESA member states (especially the PI countries: Denmark, France, Germany, Italy, Switzerland, Spain), Poland and with the participation of Russia and the USA. For this analysis we also used RXTE observations provided by the RXTE Science Operations Center and Swift/BAT transient monitor results provided by the Swift/BAT team. This work has been partially funded by the DLR, grant 50 OR 1008, and by the Carl-Zeiss-Stiftung. I.C. acknowledges financial support from the French Space Agency CNES through CNRS. We thank the anonymous referee for very valuable comments.

\section{References}

Acciari, V. A., Aliu, E., Araya, M., et al. 2011, ApJ, 733, 96

Araya, R. A., \& Harding, A. K. 1996, A\&AS, 120, 183

Basko, M. M., \& Sunyaev, R. A. 1976, MNRAS, 175, 395

Becker, P. A., Klochkov, D., Schönherr, G., et al. 2012, A\&A, 544, A123

Bradt, H. V., Rothschild, R. E., \& Swank, J. H. 1993, A\&AS, 97, 355

Caballero, I. 2009, Ph.D. Thesis, IAAT University of Tuebingen

Caballero, I., Kretschmar, P., Santangelo, A., et al. 2007, A\&A, 465, L21

Caballero, I., Santangelo, A., Kretschmar, P., et al. 2008, A\&A, 480, L17

Caballero, I., Kretschmar, P., Pottschmidt, K., et al. 2009, ATel, 2337, 1

Caballero, I., Santangelo, A., Pottschmidt, K., et al. 2010, ATel, 2541, 1

Caballero, I., Ferrigno, C., Klochkov, D., et al. 2011a, ATel, 3204, 1

Caballero, I., Kraus, U., Santangelo, A., Sasaki, M., \& Kretschmar, P. 2011b, A\&A, 526, A131

Caballero, I., Pottschmidt, K., Santangelo, A., et al. 2011c [arXiv: 1107. 3417]

Caballero, I., Müller, S., Bordas, P., et al. 2012, in AIP Conf. Ser. 1427, eds.

R. Petre, K. Mitsuda, \& L. Angelini, 300

Caballero, I., Pottschmidt, K., Marcu, D. M., et al. 2013, ApJ, 764, L23

Camero-Arranz, A., Finger, M. H., Wilson-Hodge, C., et al. 2011, ATel, 3166, 1

Ferrigno, C., Falanga, M., Bozzo, E., et al. 2011, A\&A, 532, A76

Finger, M. H., Wilson, R. B., \& Harmon, B. A. 1996, ApJ, 459, 288

Finger, M. H., Camero-Arranz, A., Kretschmar, P., Wilson, C., \& Patel, S. 2006,

in AAS/High Energy Astrophysics Division \#9, BAAS, 38, 359

Giangrande, A., Giovannelli, F., Bartolini, C., Guarnieri, A., \& Piccioni, A. 1980, A\&AS, 40, 289
Grove, J. E., Strickman, M. S., Johnson, W. N., et al. 1995, ApJ, 438, L25 Harding, A. K., \& Daugherty, J. K. 1991, ApJ, 374, 687

Isenberg, M., Lamb, D. Q., \& Wang, J. C. L. 1998, ApJ, 493, 154

Jahoda, K., Markwardt, C. B., Radeva, Y., et al. 2006, ApJS, 163, 401

Kendziorra, E., Kretschmar, P., Pan, H. C., et al. 1994, A\&A, 291, L31

Klochkov, D., Staubert, R., Santangelo, A., Rothschild, R. E., \& Ferrigno, C. 2011, A\&A, 532, A126

Klochkov, D., Doroshenko, V., Santangelo, A., et al. 2012, A\&A, 542, L28

Kraus, U., Nollert, H.-P., Ruder, H., \& Riffert, H. 1995, ApJ, 450, 763

Krimm, H. A., Barthelmy, S. D., Baumgartner, W., et al. 2009, ATel, 2336, 1

Lebrun, F., Leray, J. P., Lavocat, P., et al. 2003, A\&A, 411, L141

Li, F., Clark, G. W., Jernigan, J. G., \& Rappaport, S. 1979, ApJ, 228, 893

Lund, N., Budtz-Jørgensen, C., Westergaard, N. J., et al. 2003, A\&A, 411, L231

Makino, F., Cook, W., Grunsfeld, J., et al. 1989, IAU Circ., 4769, 1

Motch, C., Stella, L., Janot-Pacheco, E., \& Mouchet, M. 1991, ApJ, 369, 490

Mowlavi, N., Kreykenbohm, I., Shaw, S. E., et al. 2006, A\&A, 451, 187

Müller, S., Ferrigno, C., Kühnel, M., et al. 2012 [arXiv: 1211.6298]

Nagase, F., Hayakawa, S., Kunieda, H., et al. 1982, ApJ, 263, 814

Pottschmidt, K., Rothschild, R. E., Gasaway, T., Suchy, S., \& Coburn, W. 2006, in AAS/High Energy Astrophysics Division \#9, BAAS, 38, 384

Ricketts, M. J., Turner, M. J. L., Page, C. G., \& Pounds, K. A. 1975, Nature, 256, 631

Rosenberg, F. D., Eyles, C. J., Skinner, G. K., \& Willmore, A. P. 1975, Nature, 256,628

Rothschild, R. E., Blanco, P. R., Gruber, D. E., et al. 1998, ApJ, 496, 538

Rothschild, R. E., Wilms, J., Tomsick, J., et al. 2006, ApJ, 641, 801

Rothschild, R. E., Markowitz, A., Rivers, E., et al. 2011, ApJ, 733, 23

Sembay, S., Schwartz, R. A., Orwig, L. E., Dennis, B. R., \& Davies, S. R. 1990, ApJ, 351, 675

Staubert, R., Shakura, N. I., Postnov, K., et al. 2007, A\&A, 465, L25

Steele, I. A., Negueruela, I., Coe, M. J., \& Roche, P. 1998, MNRAS, 297, L5

Stella, L., White, N. E., \& Rosner, R. 1986, ApJ, 308, 669

Tchernin, C., Ferrigno, C., \& Bozzo, E. 2011, ATel, 3173, 1

Truemper, J., Pietsch, W., Reppin, C., et al. 1978, ApJ, 219, L105

Tsygankov, S. S., Lutovinov, A. A., Churazov, E. M., \& Sunyaev, R. A. 2006, MNRAS, 371, 19

Tsygankov, S. S., Lutovinov, A. A., Churazov, E. M., \& Sunyaev, R. A. 2007, Astron. Lett., 33, 368

Tsygankov, S. S., Lutovinov, A. A., \& Serber, A. V. 2010, MNRAS, 401, 1628

Tueller, J., Ajello, M., Barthelmy, S., et al. 2005, ATel, 504, 1

Ubertini, P., Lebrun, F., Di Cocco, G., et al. 2003, A\&A, 411, L131

Winkler, C., Courvoisier, T. J.-L., Di Cocco, G., et al. 2003, A\&A, 411, L1 